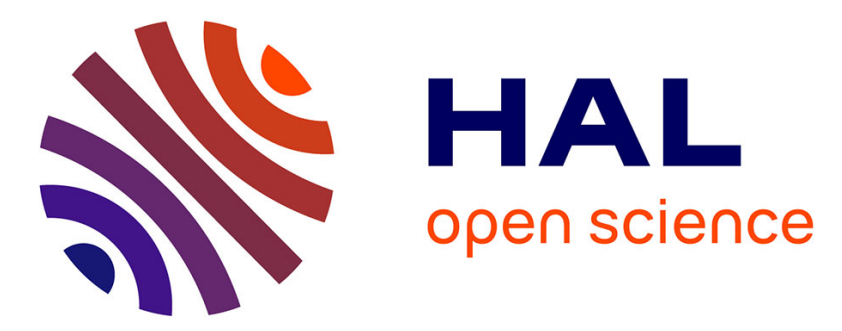

\title{
Les anciennes exploitations ferrifères des environs de Belle-Isle-en-Terre
}

\author{
Louis Chauris, J. Garreau
}

\section{To cite this version:}

Louis Chauris, J. Garreau. Les anciennes exploitations ferrifères des environs de Belle-Isle-en-Terre. Annales de Bretagne et des Pays de l'Ouest, 1990, 97 (2), pp.133-146. 10.3406/abpo.1990.3350 . insu-01860978

\section{HAL Id: insu-01860978 \\ https://hal-insu.archives-ouvertes.fr/insu-01860978}

Submitted on 5 Mar 2021

HAL is a multi-disciplinary open access archive for the deposit and dissemination of scientific research documents, whether they are published or not. The documents may come from teaching and research institutions in France or abroad, or from public or private research centers.
L'archive ouverte pluridisciplinaire $\mathbf{H A L}$, est destinée au dépôt et à la diffusion de documents scientifiques de niveau recherche, publiés ou non, émanant des établissements d'enseignement et de recherche français ou étrangers, des laboratoires publics ou privés.

\section{()ㅜ) $\Theta$}

Distributed under a Creative Commons Attribution - NoDerivatives| 4.0 International 


\section{Les anciennes exploitations ferrifères des environs de Belle-Isle-en-} Terre

Louis Chauris, Jacques Garreau

\section{Citer ce document / Cite this document :}

Chauris Louis, Garreau Jacques. Les anciennes exploitations ferrifères des environs de Belle-Isle-en-Terre. In: Annales de Bretagne et des pays de l'Ouest. Tome 97, numéro 2, 1990. pp. 133-146;

doi : https://doi.org/10.3406/abpo.1990.3350

https://www.persee.fr/doc/abpo_0399-0826_1990_num_97_2_3350

Fichier pdf généré le 04/04/2018 


\title{
Résumé
}

Les données de la toponymie, les amas de scories et les vestiges de minières, s'ajoutant aux données d'archives et aux références bibliographiques, attestent la présence d'un ancien district ferrifère aux environs de Belle-Isle-en-Terre, en Bretagne occidentale. Plus de soixante occurrences minéralisées ont pu être identifiées dans un territoire allongé d'Ouest en Est sur une vingtaine de $\mathrm{km}$. Une ancienne industrie métallurgique a été ici rendue possible par la présence de massifs forestiers relativement vastes (forêts de Beffou, de Coat-an-Noz et Coat-an-Hay) - dans une région par ailleurs le plus souvent dépourvue de bois - à proximité immédiate d'une zone ferrifère relativement étroite, offrant une juxtaposition assez exceptionnelle de différents types de gisements (sédimentaires, volcanosédimentaires, hydrothermaux, latéritiques). Les minières, toutes situées dans la zone d'oxydation, exploitaient un minerai essentiellement limonitique (éventuellement hématitique), à teneurs correctes (teneur moyenne de l'ordre de $43 \%$ de fer métal), mais très siliceux et, dans l'ensemble, de faible tonnage.

\begin{abstract}
Toponymical data, slags, traces of surface mines, archives and bibliographical data attest the presence of an old iron area near to Belle-Isle- en-Terre in Western Brittany. Over sixty occurrences have been identified in an elongated zone from west to east along about twenty $\mathrm{km}$. An. old metallurgical industry has here been possible by the presence offorests - in an area, in other respects, generally without forest - near to an iron area with different types of deposits (sedimentary, volcano-sedimentary, hydrothermal, lateritic deposits). The surface mines localized in the oxidizing zone worked an essentially limonitic (sometimes hematitic) ore, with a good grade ( $43 \% \mathrm{Fe})$, but very siliceous and, on the whole, of a low tonnage.
\end{abstract}




\title{
Les anciennes exploitations ferrifères des environs de Belle-Isle-en-Terre
}

\author{
par Louis Chauris et Jacques GARREAU
}

Les données de la toponymie, les amas de scories et les vestiges de minières, s'ajoutant aux données d'archives et aux références bibliographiques, attestent la présence d'un ancien district ferrifère aux environs de Belle-Isle-en-Terre, en Bretagne occidentale. Plus de soixante occurrences minéralisées ont pu Etre identifiées dans un territoire allongé $d^{\prime} O u e s t$ en Est sur une vingtaine de $\mathrm{km}$. Une ancienne industrie métallurgique a été ici rendue possible par la présence de massifs forestiers relativement vastes (forêts de Beffou, de Coat-an-Noz et Coat-an-Hay) dans une région par ailleurs le plus souvent dépourvue de bois - à proximité immédiate d'une zone ferrifère relativement étroite, offrant une juxtaposition assez exceptionnelle de différents types de gisements (sédimentaires, volcano-sédimentaires, hydrothermaux, latéritiques). Les minières, toutes situées dans la zone d'oxydation, exploitaient un minerai essentiellement limonitique (éventuellement hématitique), à teneurs correctes (teneur moyenne de l'ordre de $\mathbf{4 3} \%$ de fer métal), mais très siliceux et, dans l'ensemble, de faible tonnage.

Toponymical data, slags, traces of surface mines, archives and bibliographical data attest the presence of an old iron area near to Belle-Isleen-Terre in Western Brittany. Over sixty occurrences have been identified in an elongated zone from west to east along about twenty $\mathrm{km}$. An old metallurgical industry has here been possible by the presence of forests - 
in an area, in other respects, generally without forest-near to an iron area with different types of deposits (sedimentary, volcano-sedimentary, hydrothermal, lateritic deposits). The surface mines localized in the oxidizing zone worked an essentially limonitic (sometimes hematitic) ore, with a good grade (43\% Fe), but very siliceous and, on the whole, of a low tonnage.

La présence d'anciennes extractions de minerais de fer dans la région de Belle-Isle-en-Terre (Côtes-du-Nord) est en grande partie tombée dans l'oubli et le «moutonnement confus de collines $»^{1}$, jadis jalonnées de minières sur plus de $20 \mathrm{~km}$, entre le Sud de Plougras et les environs de Tréglamus, a retrouvé depuis longtemps le calme de ses forêts et de ses champs durement conquis sur les landes... La lacune, presque totale, semble-t-il, d'une documentation minière ancienne précise ${ }^{2}$, l'absence de travaux miniers en profondeur ${ }^{3}$, l'abandon séculaire des exploitations ${ }^{4}$, une structure géologique particulièrement complexe (dont les diverses cartes n'ont encore rendu compte que très imparfaitement)s, s'ajoutant à la rareté des affleurements minéralisés observables en place et à l'intense altération superficielle ${ }^{6}$ ont entraîné une certaine imprécision sur la localisation, la nature et l'origine des gisements - exploités ou non - esquissées seulement, un peu comme par hasard, à travers des publications peu nombreuses?

Actuellement, la minéralisation n'est plus, en règle générale, décelée que par des éboulis, parfois de forte dimension, plus ou moins déplacés par les coulées de solifluxion quaternaire ${ }^{8}$ ou par les épierrages et les travaux de remembrement récents. Les scories elles-mêmes ne sont plus toujours en place et ont pu être éparpillées pour l'empierrement des chemins. Quant aux installations liées aux forges elles-mêmes, elles ont presque entièrement disparu... C'est dire que l'étude du district de Belle-Isle-en-Terre a nécessité un quadrillage soigné du terrain, en vue de reconnaître la localisation des occurrences mineralisées, des lieux d'extraction et de traitement des minerais, joint à l'examen critique des données géologiques et historiques confrontées à nos nouvelles recherches. Les apports de la toponymie et les enquêtes auprès des cultivateurs' se sont souvent révélés précieux.

Le présent mémoire doit être considéré comme une vue d'ensemble préliminaire de l'ancien district ferrifère de Belle-Isle-en-Terre. D'aucuns s'étonneront peut-être de voir parfois l'« Histoire naturelle » privilégiée aux dépens de l'« Histoire tout court ». C'est que, dans le cas examiné ici, la première commande fondamentalement la seconde : sans cette connaissance de base, les hypothèses historiques risquent de se retrouver bientôt sans fondement. Par ailleurs, les recherches approfondies que demandera encore l'approche historique, ne manqueront pas d'être facilitées par la pré- 
sente étude. Peut-être cet essai incitera-t-il aussi à d'autres travaux sur divers districts ferriferes de Basse-Bretagne, encore très mal connus ${ }^{10}$ et dont les derniers vestiges s'estompent rapidement.

\section{Données de la toponymie}

Les toponymes indiquant la présence de minerais de fer et la métallurgie de ce métal sont encore relativement nombreux dans le district de BelleIsle-en-Terre, tant en langue bretonne qu'en langue française (fig. 1). A titre d'exemple, on citera : Les Ferrières, Quinquis-Ferrières, les Ferrières Izellan, en Plougonver ; Roz an Goff (Tertre du forgeron) en Loc-Envel ; Roscoff (même signification) en Loguivy-Plougras ${ }^{11}$; le Gouelou (les Forges) ${ }^{12}$ également en Loguivy-Plougras; les Vieilles Forges en BelleIsle-en-Terre ; les Forges de Coat-an-Noz (Belle-Isle); les Forges (un peu au sud de Belle-Isle); la Fonderie... Il est évident que la micro-toponymie (étude des noms des différentes parcelles du cadastre) apporterait d'autres indications précieuses $(13,14)$. L'ancienneté - relative - de plusieurs de ces toponymes est attestée par leur présence sur la carte de Cassini (Les Ferrière (sic) ; Rosengoff ; Forge de Coatannos (sic)). Actuellement, divers toponymes qui ne correspondent plus à aucune réalite, tendent à disparaître. Cette évolution apparaît clairement en comparant la carte d'Étatmajor à 1/80000 (édition de la fin du $\mathrm{XIX}^{\circ}$ siècle) avec les nouvelles cartes à $1 / 25000$ de 1'I.G.N. ${ }^{15}$.

\section{Amas de scories}

Un autre signe tangible - très classique - d'une activité métallurgique ancienne, se manifeste par la présence d'accumulations de scories. Ces résidus du traitement des minerais de fer sont bien reconnaissables par leur teinte (le plus souvent brunâtre ou gris noirâtre), leur texture interne bulleuse et leur surface riche en bourrelets cordés ${ }^{16}$. Des amas de scories ont déjà été signalés par Ch. Barrois ${ }^{17}$, F. Kerforne ${ }^{18}$, L. Puzenat ${ }^{19}$, M. Gouin ${ }^{20}$ et B. Mulot ${ }^{21}$ aux lieux-dits Pengalet, le Rocher, Landeven, Kerbalanen, Kerambley, Lestrezen, Treusvern, Guinaman, Gars al Louět, Loquervezen, les Ferrières..., ainsi que dans la forêt de Beffou et aux forges de Coat-anNoz (fig. 1). Ces vestiges disparaissent peu à peu ; ils sont utilisés en particulier pour l'empierrement des chemins ruraux.

Au cours de nos explorations, plusieurs tas de scories, localement encore assez importants, ont pu être observés (fig. 1). Au Gouelou (en LoguivyPlougras), ils sont situés un peu au nord du village (en grande partie ruiné), à la limite méridionale de la forêt de Beffou ; l'échancrure pratiquée en vue 
de l'empierrement permet de recueillir, au milieu des scories, quelques fragments de minerai de fer. A l'ouest de Cosqueric, à la lisière orientale de la même forêt, un amas de scories a été mis à jour par l'ouragan du 16 octobre 1987, qui a déraciné les grands arbres masquant le dépôt. A Trovern, à près d'1,5 km de la limite occidentale actuelle de la forêt de Beffou, les scories forment, immédiatement au Nord du hameau, en grande partie abandonné, plusieurs tas surbaissés (environ deux mètres de haut) envahis par une végétation arborescente...

Dans l'état actuel des recherches, il n'a pas encore été possible de dater les accumulations de scories ${ }^{22}$. Leur ancienneté est suggérée par l'ampleur prise par la végétation qui les voile le plus souvent au regard. L'absence totale de cours d'eau à leur voisinage indique qu'elles représentent les résidus de forges à bras, autre marque d'ancienneté ${ }^{23}$. En l'absence de sites de grosses forges, ces nombreuses occurences de scories témoignent ainsi de la présence ancienne de bas fourneaux. Le report sur la carte (fig. 1) montre que ces établissements dessinent une sorte d'arc de cercle vers la bordure méridionale de l'actuelle forêt de Beffou et vers ses anciens prolongements présumés ouest et est, établissant ainsi la provenance toute proche du charbon de bois. L'origine du minerai de fer était également proximale (infra et fig. 2). Le rendement du traitement du minerai paraît avoir été assez médiocre, ainsi que l'atteste, en l'absence d'analyses chimiques, le pourcentage élevé des petits fragments attirés par l'aimant. En fait, il existe ici - comme partout - tous les intermédiaires entre les scories sensu stricto, denses, encore riches en fer (très abondantes) et le laitier proprement dit, léger, très pauvre en métal (beaucoup plus rare) ${ }^{24}$.

\section{Aspects actuels des anciennes extractions}

D'après les vestiges encore visibles, les exploitations ferrifères du district de Belle-Isle-en-Terre ont été individuellement peu importantes, sont restées généralement disséminées, mais au total ont été relativement nombreuses. Cette manière de voir est en bon accord avec les observations de l'Ingénieur des Mines qui parcourut la région aux alentours de $1840^{25}$ : " les gîtes... sont peu abondants, assez éloignés les uns des autres, les exploitations s'ouvrent irrégulièrement, suivant le caprice ou l'instinct des mineurs. Elles consistent généralement en petits puits dont la profondeur maximum est de 12 mètres ».

Les vestiges des extractions à ciel ouvert - ou minières - sont actuellement de trois types.

- Toute trace directe de la minière a disparu par comblement' ${ }^{26}$. La présence d'une ancienne exploitation est cependant fortement suggérée par des 
FIG. 1

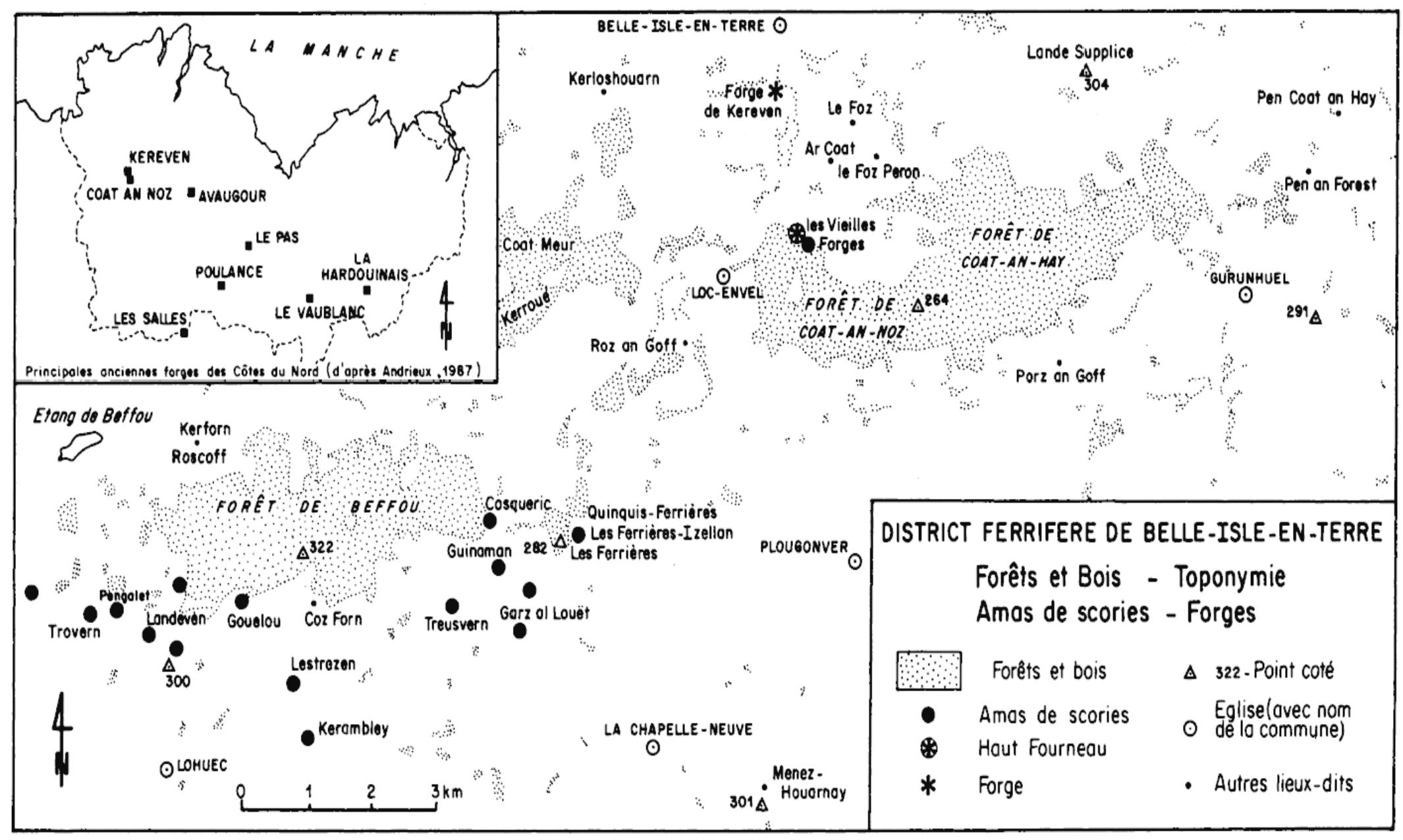


fragments de minerai de fer plus ou moins abondants, épars dans les champs à présent cultivés, ou, plus souvent, rassemblés à la bordure des parcelles, lors des épierrages, quand ils n'ont pas été dispersés ultérieurement aux alentours pour l'empierrement des chemins. Ce premier type est très répandu. Dans certains cas limites, il n'y a même pas eu d'ouverture de minière proprement dite. Un document ancien ${ }^{27}$ semble bien indiquer que pendant plusieurs années, seul le minerai épars à la surface du sol était exploité (pour le haut fourneau de Coat-an-Noz): "Il n'y a aucunes minières anciennes (sic) ouvertes, la mine n'est que volante depuis quelques années ». Dans ce cas, il s'agit plus d'un grappillage superficiel (qui s'interrompt sans laisser de traces) que d'une exploitation minière au sens strict.

- La trace de la minière se marque encore par une concavité du terrain plus ou moins accusée. La minière a été comblée, mais le tassement des remblais provoque peu à peu le développement d'une sorte de cuvette très surbaissée. Un tel dispositif était encore visible aux Ferrières en $1960^{28}$. Il a été reconnu récemment (1989) près de Colledennou en Tréglamus, au milieu d'un champ résultant du remembrement de 16 parcelles ; les fragments de minerai épars sont encore abondants. Il est très possible que les irrégularités topographiques décelées près de Coat Treusvern représentent également les traces d'une ancienne minière ; les blocs de minerai sont ici très nombreux.

- La minière est encore bien visible, bien que sa partie inférieure soit noyée et que ses flancs soient envahis par une végétation luxuriante. Sauf erreur d'interprétation, un bon exemple est toujours observable à midistance entre le Heyou et la cote 170, au Nord - Nord-Est des Vieilles Forges de Coat-an-Noz. L'excavation, étroite mais très allongée, est localement muréc vcrticalement (sans doute pour éviter les éboulements lors des travaux d'approfondissement) ${ }^{29}$.

\section{Localisation des minières et des indices ferrifères}

Plus de soixante dix occurrences de minerai de fer (anciennes minières et indices ferriferes dont l'exploitation - fort possible - n'est cependant pas toujours attestée avec certitude) ont été recensées dans le district de Belle-Isle-en-Terre. La figure 2 fournit pour la première fois une représentation cartographique précise du district dont la signification gîtologique et économique (sous l'angle historique) est ainsi nettement établie ${ }^{30}$.

Cette carte, qui constitue l'un des apports fondamentaux de notre travail, appelle un certain nombre de remarques.

- Les documents anciens confirment la fréquence des « mines », mais, en même temps, en règle générale, leur petite taille. Ainsi le rapporteur sur 
FIG. 2

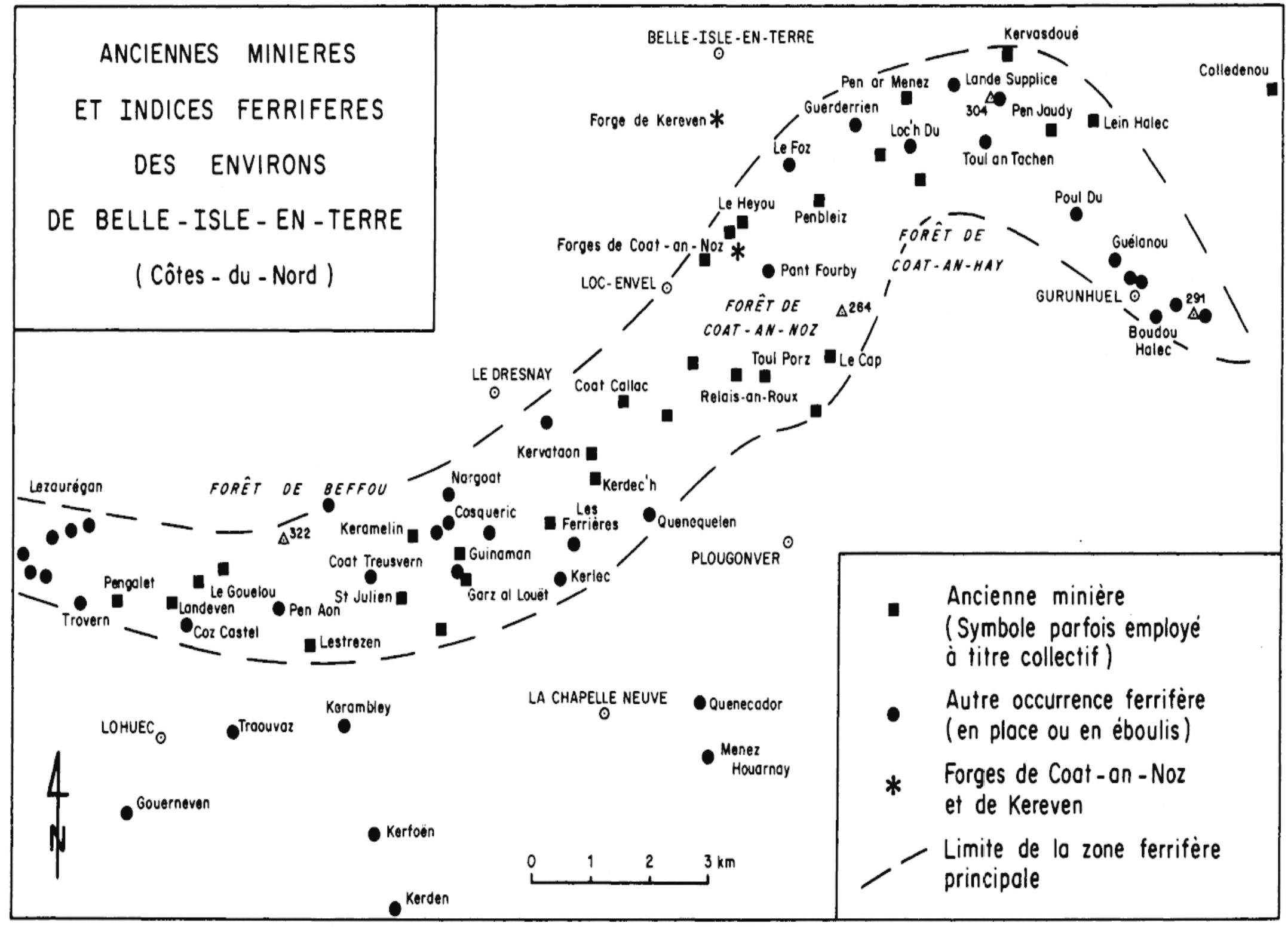


l'état des forges de Coat-an-Noz en $1783^{31}$ écrit : "les mines sont ici on ne peut pas plus communes et fort près du fourneau de fusion » et il ajoute « nos mineurs nous en ont tiré depuis la création de notre établissement environ 12 mil barriques ». Les réserves sont considérées comme importantes par le même rapport : « la mine étant très abondante dans le pays et susceptible de ne pas manquer de plusieurs siècles». Toutefois, d'autres documents font douter de la « richesse » minière des environs de Coat-anNoz : tel le témoignage rapporté par Andrieux ${ }^{32}$ : «Accrédités pour tirer la mine jusqu'à deux lieues à la ronde de leur fourneau, les industriels de Coat-an-Noz épuisèrent bientôt les petits affleurements qui parsemaient le sous-bois et durent demander à étendre leurs recherches jusqu'à quatre lieues ». Cette remarque, qui s'accorde bien avec les annotations de De Fourcy ${ }^{33}$, est confirmée par la lecture des documents d'archives ${ }^{34}$. Citons un peu au hasard, quelques phrases révélatrices :... « le peu de mine que l'on était parvenu à ramasser... " "Quand les filons de mine ne se perdent pas... » (lors des travaux d'approfondissement). ...«Il a été fait des milliers de fouilles sans avoir trouvé aucun foyer à produire facilement l'approvisionnement de trois mois du fourneau... »...«L'endroit où l'on travaille en ce moment n'offre pas encore de grandes espérances... ». La lettre du directeur de l'usine Coat-an-Noz au préfet des Côtes-du-Nord (en 1840 ou 1841 ? $)^{35}$ en vue d'obtenir l'autorisation d'extraire du minerai de fer sur la ferme de Pen ar Menez en Louargat témoigne de la nécessité d'un approvisonnement en minerai au-delà des abords immédiats du haut fourneau. Par ailleurs, l'eau constituait un obstacle majeur à la poursuite des travaux $^{34}$ : «l'on est qu'à 18 pieds de profondeur et il faut tirer de l'eau... » ...« A 20 pieds de profondeur, l'on est arrêté par l'eau... ». En 1783², les forges de Coat-an-Noz employaient 12 mineurs sur un total de 143 personnes.

- Les travaux géologiques de nos prédécesseurs sont surtout précieux par les indications fournies sur les sites exploités dans le passé ou sur les occurrences mises en évidence au cours de leurs levers sur le terrain. Toutefois, en l'absence - le plus souvent - de carte, les localisations précises ont pu parfois s'avérer délicates. En outre, plusieurs publications ne font en fait que reproduire, sans vérification, les acquis antérieurs, et ne sont qu'une simple succession de noms, sans commentaire.

- Plusieurs occurrences ne sont pas observées « en place », mais ont été déplacées (a) soit par l'homme, lors des opérations de remembrement; le déplacement est alors, généralement, assez faible et se tient dans les limites des parcelles (accumulation en bordure); (b) soit par les processus de solifluxion quaternaire ${ }^{36}$. Ainsi, une incertitude demeure sur la position originelle de nombreux blocs de quartz ferrugineux notés entre Cosqueric et Nargoat, vers la base du large glacis qui s'étale au Nord-Ouest de 
Loquervezen (près d'une centaine de mètres de dénivelé). Il en est de même pour les fréquents éboulis ferrifères observés sur les pentes de la Lande Supplice (Menez Hoguéné). Quoiqu'il en soit, en place ou pas, ces dernières occurrences ont sans doute été jadis exploitées du fait de la belle qualité du minerai et de la proximité des gisements de fer de Pen ar $\mathrm{Menez}^{37}$ et de Kervasdoué.

- La zone minéralisée principale (65 occurrences) est essentiellement comprise dans une bande allongée, légèrement supérieure à une vingtaine de $\mathbf{k m}$, qui s'étire d'ouest en est sous forme d'une sorte d'S couché, d'un à trois $\mathrm{km}$ de large. Les rares autres occurrences (au nombre de sept) sont disséminées plus au Sud, sans ordre apparent. La zone majeure peut, à son tour, être divisée en deux parties : une partie occidentale qui dessine une sorte de lunule au Sud-Ouest, au Sud et au Sud-Est de la forêt de Beffou ; une partie orientale, qui forme également une lunule, mais de courbure opposée à l'ouest, au nord et à l'est de forêts de Coat-an-Noz et de Coat-anHay.

- Le rapprochement de la figure 2 avec la figure 1 (où sont reportés les amas de scories) suggère vivement que la partie occidentale a jadis alimenté des bas fourneaux (supra), tandis que la partie orientale a principalement alimenté le haut-fourneau, plus récent, de Coat-an-Noz. Ainsi, pour la première fois dans le district ferrifère de Belle-Isle-en-Terre est mise en évidence une certaine "logique du paysage minier et métallurgique „38. On ignore encore si les nombreuses occurrences des environs immédiats de Gurunhuel, situées à l'extrémité du district ferrifère, ont été exploitées. Il est évident que l'augmentation des distances entre les lieux d'extraction et de traitement des minerais constituait alors un handicap très sérieux.

\section{Nature et origine des minerais de fer}

Avant de conclure, il s'avère nécessaire de préciser la nature et l'origine des minerais de fer du district de Belle-Isle-en-Terre. Dans le passé, ces données ont, en effet, conditionné les modalités de l'extraction, l'importance relative des gîtes et, enfin, le traitement métallurgique. A l'avenir, seules de telles observations permettent de fournir une base solide à l'analyse des scories. Sans entrer dans les détails pétrographiques et chimiques qui n'ont pas leur place ici, les résultats obtenus sont brièvement synthétisés.

Rappelons d'abord que les documents anciens ne fournissement pas d'indications précises sur la nature des minerais exploités. Les données d'archives soulignent toutefois la variété des minerais traités ${ }^{39}$. "La qualité des mines extraites à Coat-an-Noz... est en général et sans contredit une 
des bonnes de la Province. L'une est une mine de couleur violette extraordinairement compacte... donnant des fers d'une mediocre qualité mais rendant plus que toutes les autres. L'autre est jaunâtre... légère... rendant peu... ». Le troisième minerai est appelé « mine creuse ou sabotteuse ». Et l'auteur de suggérer un mélange soigneux de «ces trois espèces de mines » pour donner une « fonte délicieuse »...

Le tonnage total des minerais extraits dans le district ferrifere de BelleIsle-en-Terre est impossible à évaluer, même d'une manière approximative. Toutefois, quelques données chiffrées permettent parfois de se faire une idée des extractions annuelles sur l'ensemble du district, voire sur la production d'une minière. Pour 1789, le tonnage de minerai extrait n'est pas connu, mais la production de fonte à Coat-an-Noz s'élève à 3000 quintaux métriques ${ }^{40}$. En 1813, la quantité de minerai consommé par le haut fourneau de Coat-an-Noz est de 1000 q.m. ; pour les 9 premiers mois de 1814, elle s'élève à 1332 q.m. ${ }^{40}$. En $1842^{41}$, les minières de Coat-an-Noz avaient produit 14000 q.m. de minerai, débourbé sur place. En 1823 (?), la production de la minière de la Lande des Partentiers dans la commune de Belle-Isle est estimée seulement à 80 (q.m. ?) ${ }^{40}$.

Les minerais exploités étaient essentiellement situés dans la «zone d'oxydation $\gg^{42}$. En conséquence, ils sont surtout de composition limonitique $^{43}$; certaines zones plus profondes renferment de l'hématite ${ }^{44}$. Les teneurs en fer métal, sans être très élevées, sont satisfaisantes. Nos analyses (11 échantillons) ${ }^{45}$ ont donné des valeurs comprises entre $33 \%$ (Lein Halec) et $56 \%$ (Quenequelen) et une teneur moyenne de $43,2 \%$. La principale caractéristique des minerais est leur composition fortement siliceuse (teneur moyenne en $\mathrm{SiO}_{2}=26 \%$, avec les extrêmes compris entre 4,6 et $44,9 \%)^{46}$. Les teneurs en alumine $\left(\mathrm{Al}_{2} \mathrm{O}_{3}\right)$ sont également très variables (de $<1 \%$ a $11,8 \%$ ); teneur moyenne $=6 \%$. Les teneurs en oxyde de manganèse sont très basses $(\mathrm{MnO}=0,04 \%)$. La teneur moyenne en $\mathrm{P}_{2} \mathrm{O}_{5}$ est de $0,41 \%$. Les teneurs en oxyde de titane sont differentes suivant les minerais $\left(\mathrm{TiO}_{2}\right.$ va de $0,02 \%$ a $1,57 \%$ ). Ces dernières variations, ainsi que les variations des teneurs en vanadium, chrome, nickel, baryum... s'avèrent fort utiles pour préciser l'origine première des diverses occurrences ferrifères du district de Belle-Isle-en-Terre.

Indépendamment des processus d'oxydation superficielle envisagés dans le paragraphe précédent, les minerais du district de Belle-Isle-en-Terre peuvent présenter quatre origines primaires différentes selon les gisements :

- sédimentaire, en relation avec la sédimentation dans la mer dévonienne ;

- volcano-sédimentaire, en liaison avec des processus d'exhalaisons sous-marines associées au volcanisme dévonien ; le minerai primitif était 
ici essentiellement sulfure (pyrite); seul l'indice de Gouerneven correspond à ce type (d'après la notice de la feuille géologique «Belle-Isle-enTerre » op. cit., notes ${ }^{30.47}$;

- hydrothermale, en relation avec les filons quartzeux formés dans des zones de dislocation consécutives aux mouvements hercyniens; ici, le minerai était sans doute primitivement carbonaté (sidérite) ;

- latéritique, en liaison avec les climats tropicaux de l'époque tertiaire, avec formation d'une cuirasse aux dépens de diverses roches déjà riches en fer (dolérites, amphibolites, serpentines...) ${ }^{48}$.

\section{Conclusions}

Au point de vue historique des exploitations minières et du traitement sidérurgique, le district de Belle-Isle-en-Terre paraît pouvoir être subdivisé, schématiquement, en deux parties :

- un sous-district occidental - ou district de Beffou-jalonné par des exploitations anciennes, ayant alimenté des bas fourneaux d'importance très subordonnée ; ici, les cours d'eau font pratiquement défaut. L'archéologie minière est susceptible de se révêler particulièrement prometteuse dans ce secteur encore très mal connu ;

- un sous-district oriental - ou district de Coat-an-Noz - avec la présence d'exploitations plus récentes, alimentant à la fin du XVIII siècle et pendant la première partie du $\mathrm{XIX}^{\boldsymbol{e}}$ siècle, un haut fourneau relativement important (Coat-an-Noz), situé au bord d'un cours d'eau assurant la force motrice ${ }^{49}$.

L'intérêt économique présenté dans le passé par le sous-district de Coatan-Noz résidait essentiellement dans sa proximité relative du port de guerre de Brest. A la fin du XVII' siècle, les forges de Coat-an-Noz pouvaient ainsi fournir au grand port du Ponant, boulets, lest et autres objets en fer ${ }^{\text {so }}$.

\section{NOTES}

1. E. de Martonne. France physique, p. 80 , Edit. A. Colin, Paris, 1947, 464 p. La forêt de Beffou culmine à $322 \mathrm{~m}$; la forêt de Coat-an-Noz, à $264 \mathrm{~m}$; les hauteurs de Gurunhuel à $304 \mathrm{~m}$; à Belle-Isle-en-Terre, Le Léguer coule à moins de 100 mètres.

2. Les seuls « savants * ayant publié leurs observations sur les gisements de Belle-Isle-
en-Terre au moment de lew exploitation sont, à notre connaissance, Gillet de Laumont (Inspecteur général des Mines de France) dans sa $\propto$ Description de plusieurs filons métallifères de Bretagne.... Joumal de Physique, 22 p., 1786, et E. de Fourcy (Ingénieur en Chef des Mines) dans son 
mémoire sur la « Carte géologique des Côtes-du-Nord », Edit. Fain et Thunot, Paris, 172 p., 1844. Les archives conservent des données intéressantes sur les forges de Coat-an-Noz (voir en particulier le remarquable mémoire de J.Y. Andrieux « Forges et hauts foumeaux en Bretagne du Xvir au $x_{1} x^{e}$ siècle) ». Edit. Cid, Nantes, 328 p., qui consacre plusieurs pages aux forges de Coat-an-Noz), mais fort peu de documents sur les exploitations minières elles-mêmes (A.D. Ille-et-Vilaine C 1475 ; A.D. Côtesdu-Nord, Série S : 80 S2 (134), 6M 937...).

3. Au maximum une douzaine de mètres, selon de Fourcy, op. cit. note 2. La profondeur de la grande minière probable, partiellement encore non comblée, visible un peu à l'ouest du Heyou, au sud de Belle-Isle, paraît être du même ordre de grandeur.

4. La date précise de l'arrêt des demières exploitations n'a pu être déterminée. Selon M. Gouin « Synthèse des connaissances acquises sur les minerais de fer du département des Côtes-du-Nord : Rapport B.R.G.M. inédit DSGR. 66 A7, 39 p., 1966, la dernière minière en activité a été celle des « Forges » (il s'agit sans doute du site du Heyou, cité note 3). Logiquement, l'extraction n'a pu survivre a l'arrêt du hautfoumeau de Coat-an-Noz en 1843.

5. A ce sujet, on comparera avec intérêt les deux éditions successives de la carte géologique a 1/80 000 feuille * Morlaix * (Edit. de 1905, due à Ch. Barrois et édit. de 1962, due à $\mathrm{Ch}$. Delattre et P. Pruvost) et la récente édition de la carte à $1 / 50000$. feuille Belle-Isle-en-Terre, coordonnateur J. Chantraine, 1984).

6. Les climats successifs qui ont affecte la Bretagne ont profondément décomposé le sous-sol. A l'intense altération due au climat tropical tertiaire ( la maladie tertiaire " d'Y. Milon) se sont superposées les différentes périodes glaciaires quatemaires, avec leurs altemances de gel et de dégel, provoquant d'importants phénomènes périglaciaires de gélifraction (fracturation des roches), suivis de solifluxion (déplacement par écoulement lent des fragments gélifractés le long des pentes).

7. Les références complémentaires aux indications reportées dans la note 2 seront présentées plus loin.

8. Voir note 6 . En plusieurs points de Bretagne, il a pu être établi que le transport de blocs pierreux lié à la solifluxion pouvait atteindre plusieurs centaines de mètres, voire le kilomètres, même sur des pentes de quelques degrés seulement.

9. Aucun agriculteur encore en vie n'a évidemment connu les périodes d'activité minière et métallurgique. Mais la connaissance de leur domaine les rend irremplacables dans la recherche de minières comblées ou des amas de scories masqués dans les bois.

10. Nous pensons ici plus particulièrement aux (très) anciennes exploitations du Finistère (presqu'lle de Crozon et environs du Faou, districts de Châteaulin, Briec, Braspart, Huelgoat ; plusieurs points entre Morlaix et Landerneau). Voir L. Puzenat (1939). La siderurgie armoricaine. Mém. Soc. géol. minéral. Bretagne, 400 p. : M. Gouin (1966). Synthèse des connaissances acquises sur les minerais de fer du Finistère. Rapport B.R.G.M., inédit, DSGR 66 - A6, 39 p. Un contraste frappant apparaît a ce point de vue avec les districts plus orientaux de la Bretagne et avec les pays de la Loire. A ce sujet, on citera le remarquable ouvrage * Les forges du Pays de Châteaubriant * paru dans les Cahiers de Inventaire, en 1984 sous la direction de J.F. Belhoste et H. Maheux ; le travail de F. Dornic * Le fer contre la forêt $\%$, Edit. Ouest-France, 1984, 256 p., et le très intéressant mémoire de J.Y. Andrieux, op. cit., note 2. Voir aussi J.C. Meuret (1989). Le paysage métallurgique et minier ancien du sud-est de l'llle-etVilaine, Ann. de Bretagne, t. 96, 2, p. 145 159.

11. Le toponyme « Porz an Goff en Plougonver est d'interprétation plus délicate. S'agit-il de la $*$ ferme du forgeron $»$ ou tout simplement de la « ferme de Le Goff * (anthroponyme) ?

12. Normalement, la forge s'écrit « govel $\star$. Il s'agit ici d'une déformation dialectale locale.

13. Quelques toponymes restent difficiles a interpréter. Ainsi * Menez Houamay * en la Chapelle-Neuve doit-il se traduire par * Montagne du fer » (mais alors on aurait plutôt attendu Menez Houarneg) (on notera que nous avons decouvert du minerai de fer a Menez-Houamay) ou par la * Montagne de (Saint) - Herve * (variante d'Houarneau). Ce menez qui atteint 301 mètres serait alors un équivalent, un peu plus méridional, du Mené Bré (Également 301 mètres) avec sa Chapelle Saint-Herve. De même, * Coz. Forn " en Lohuec, au Sud de la forêt de Beffou, en plein district ferrifere, peut-il s'inter- 
préter comme « vieux four » (de forgeron) ou (à pain)? Dans le cas présent, le contexte privilègerait plutôt la première hypothèse. Même remarque pour « Kerforn » à l'Est de l'étang de Beffou ; la présence d'un « Roscoff » (avec amas de scories) à proximité est ici aussi en faveur d'un four de forge. Le toponyme \& Foz» pose également problème : faut-il entendre « la fosse », au sens dancienne excavation du type minière? (le Foz; le Foz Peron au Sud de Belle-Isle). La découverte de minerai de fer à proximité du lieu-dit le Foz peut appuyer une telle interprétation. Peut-être cependant s'agit-il des « fossés » d'un ancien château? Quant à Kerloshouarn » il ne s'agit évidemment pas d'un * Kerlost Louarn $»$ imaginaire (hameau du fenouil sauvage) mais d'un dérivé de l'anthroponyme Vieux-Breton \& Loies Hoiarn » (B. Tanguy, comm. orale). La forge de Kereven était une petite usine de seconde fusion, installee sur le Guic; sa période d'activité s'est étalée entre 1828 et les environs de 1860 .

14. L'observation sur le terrain s'impose ici (comme partout!) pour vérifier les témoignages. Les cultivateurs des environs de Belle-Isle-en-Terre, comme de beaucoup d'autres régions de Basse-Bretagne, désignent souvent sous le nom de * mein houarn $\star$ ( $*$ pierre de fer ${ }^{\circ}$ ) des roches dures, denses et sombres (par exemple des « dolérites $»)$ qui ne sont aucunement des minerais de fer!

15. Outre les minerais de fer, la région de Belle-Isle-en-Terre renferme également des minerais plombifères qui ont fait l'objet d'exploitation au Xviur siècle. D'anciens vestiges sont encore visibles dans la forêt de Coat-an-Noz, en particulier près du lieu-dit - Toul Plom * (le trou du plomb). C'est sans doute vers la même période qu'ont été mis en évidence, toujours dans la forêt de Coatan-Noz, des indices de tungstène et de cuivre: une vieille galerie est encore connue sous le nom de * Toul al lutun $\$$ (le trou du lutin) (voir L. Chauris (1957). Presence de wolfram à Coat-an-Noz, près de Belle-Isle-en-Terre (Côtes-du-Nord). C.R. Acad. Sciences, Paris, t. 245, p. 2331 -2333 : Y. Lulzac (1963). La région minéraliste de Coat-an-Noz. Etude préliminaire. Rapport B.R.G.M. inédit VB 225, 32 p. 5 pl. h.t. : Y. Lulzac et L. Chauris, à paraître.

16. Connus en breton sous le nom evocateur de $*$ Koc'h houarn $\star(m . .$. de fer $!)$.
17. Carte géologique... op. cit. note 5.

18. Contribution à l'étude des minerais de fer du département des Côtes-du-Nord. Bull. Soc. géol. minéral. Bretagne, 1920, I, p. 292-332.

19. La sidérurgie armoricaine..., op. cit. note 10.

20. Synthèse des connaissances..., op. cit. note 4.

21. Atlas-Guide des anciennes minières et mines de fer dans le département des Côtesdu-Nord. Rapport inedit, 1971, 37 p.

22. Sauf, Evidemment, celles des forges de Coat-an-Noz, liées a l'activité du haut fourneau (fin du Xvur siecle-première moitie du XIX siècle).

23. Par rapport aux forges de Coat-an-Noz, sises au bord d'un cours d'eau. Voir J.Y. Andrieux, op. cit., note 10.

24. On consultera avec fruit la synthèse très claire de F.H. Forestier sur l'ancienne métal lurgie du fer, présentée dans l'annexe de l'intéressant mémoire de cet auteur sur les * apports récents de la géologie, de l'archéologie et de la microtoponymie à la localisation d'activités industrielles anciennes sur le plateau de Craponne-sur-Arzon. Fonderies et exploitations minières $\%$. Extrait des « Cahiers de la Haute-Loire », 1985, 70 p., 2 cartes h.t. Pour plus de détail, voir aussi Tylecote R.F (1976) \& A history of metallurgy *. The metals Society edit., et Marechal J.P. (1983). * La préhistoire de la metallurgie et ses prolongements 20 . Revue archéologique. Sites, hors-série, $n^{\circ} 14$.

25. E. de Fourcy, Carte géologique... op. cit. note 2

26. Ou n'a pu être retrouvée - ce qui pourrait s'expliquer dans le cas de petits puits, présent masqués par la végétation.

27. A.D. Côtes-du-Nord 6 M 937.

28. Observation d'un des auteurs (L.C.), en compagnie de J. Bouladon, ingénieur des mines de Paris.

29. La confusion des anciennes minières avec les vieilles ardoisières, comme celles jadis exploitées a Trovern vers l'extrémite occidentale du district ferrifere, est exclue du fait de la nature des déblais. Les nombreux déblais anciens situés près du Château de Coat-an-Noz se rapportent aux travaux miniers sur les occurrences plombifères. Voir Lulzac et Lulzac et Chauris, op. cil., note 15.

30. La carte a eté dressé d'après les travaux de E. de Fourcy (op. cit. note 2) : Kerforne (op. cit. note 18); Puzenat (op. cit., 
note 10); Gouin (op. cit., note 4); Mulot (op. cit., note 21) ; Delattre Ch., Pruvost P. et Waterlot G. (1957). Briovérien et Dévonien au Sud de Belle-Isle-en-Terre (feuille de Morlaix, au 1/80 000), Bull. Serv. Carte géol. France, $n^{\circ} 252$, t. 55 , p. 57-62; Lulzac, 1963, op. cit. note 15 ; J.C. Picot : * Indices de scheelite liés à des roches basiques en Basse-Bretagne $»$, Thèse $3^{e}$ cycle, Paris, 1975,220 p. ; la notice de la carte géologique Belle-Isle-en-Terre au 1/50 000 edit. BRGM, 1984, et nos observations personnelles.

31. Arch. départ. Ille-et-Vilaine C 1475.

32. Forges et hauts foumeaux... op. cit. note 10 .

33. Carte géologique... op. cit. note 2 .

34. A.D. Côtes-du-Nord 6 M 937.

35. A.D. Côtes-du-Nord 80 S2 134.

36. Notes 6 et 8 .

37. A.D. Côtes-du-Nord, série S, 80 S2 (134).

38. J.Y. Andrieux et A. Brule (1989) * Une histoire métallưgique et minière inscrite sur le terrain ». Ann. de Bretagne, t. 96, 2, p. $115-122$.

39. A.D. Ille-et-Vilaine C 1475.

40. A.D. Côtes-du-Nord 6 M 337.

41. De Fourcy, 1844, op. cit. note 2.

42. Partie superficielle des gisements métallifères, soumise a l'altération météorique par les infiltrations d'eaux de pluie.

43. La limonite est un mélange d'hydroxydes de fer où domine la goethite.

44. Oxyde de fer de formule $\mathrm{Fe}_{3} \mathrm{O}_{3}$. La couleur de la poussière permet de distinguer facilement la limonite (poussière jaune brunâtre) de l'hématite (poussière rouge sang à rouge brun).

45. Effectuées au Bureau de Recherches Géologiques et Minières, Orléans.
46. Cette teneur élevée en silice représentait un handicap sérieux. En effet, au cours des opérations de réduction, FeO a tendance à se combiner avec $\mathrm{SiO}_{2}$ pour former un silicate de fer (la fayalite), diminuant d'autant le pourcentage en métal obtenu. Voir Forestier, op. cit. note 24.

47. Les limonites superficielles (ou « chapeau de fer $»)$ de ces amas sulfurés peuvent masquer la présence en profondeur de gisements semblables a celui mis en évidence par le B.R.G.M. à Bodennec, un peu à l'Ouest de la région étudiée ici. Voir F. Aye, 1983 : * Contrôles géologiques des gîtes stratiformes de $\mathrm{Pb}, \mathrm{Zn}, \mathrm{Cu}, \mathrm{Ag}$ de la bordure du bassin de Châteaulin (Massif armoricain, France). Mém. BRGM n० 120, 256 p.

48. La distinction de ces quatre types differents repose sur des observations de terrain, appuyes par des examens au microscope et des analyses chimiques. Ces problèmes génétiques feront lobjet d'une note la * Chronique de la Recherche minière $₫$.

49. Cette constation n'implique évidemment pas l'absence, ici, d'une $\propto$ industrie ferrifère antérieure. Inversement, il est certain que les minières les plus orientales du sous-district occidental ont été réexploitées pour le haut foumeau de Coat-an-Noz. 50. Arch. Nat. F. 144333 (3) « La forge de Coat-an-Noz, situé à 20 lieues de Brest, par la facilite de son transport, par terre et par mer, peut être du plus grand avantage au port de Brest et lui foumir lest, boulets, et autres $»$.

Remerciements. Les figures ont été redessinés par J. L. Travers ; le texte dactylographie par M.P. Schutz. 